\title{
Individualised behavioural counselling for smoking mothers decreased children's exposure to smoke
}

\author{
Hovell MF, Zakarian JM, Matt GE, et al. Effect of counselling mothers on their children's exposure to environmental \\ tobacco smoke: randomised controlled trial. BMJ 2000 Aug 5;321:337-42.

\section{QUESTION: Does individualised behavioural counselling for smoking mothers reduce children's exposure to environmental tobacco smoke (ETS)?}

\section{Design}

Randomised (allocation not concealed), blinded (outcome assessors) controlled trial with follow up at 1 year.

\section{Setting}

San Diego county, California, USA.

\section{Participants}

108 English and Spanish speaking mothers (mean age 29 y; $47 \%$ white, $28 \%$ Hispanic, $21 \%$ black), who smoked $\geqslant 2$ cigarettes each day and exposed their child $(<4 \mathrm{y}$ of age) to the smoke from $\geqslant 1$ cigarette each day, were identified from service sites of the supplemental nutrition programme for women, infants, and children. Women who were breast feeding and those who did not have a telephone were excluded. Follow up was $\geqslant 87 \%$.

\section{Intervention}

53 mothers were allocated to individualised behavioural counselling, which comprised 3 in person and 4 telephone counselling sessions over a 3 month period. Sessions were done by trained graduate students and focused on shaping procedures (ie, complex smoking practices were gradually altered to reduce the child's exposure to ETS). During sessions, mothers set long term goals, signed contracts, and wrote objectives twice a month; counsellors reviewed progress and negotiated possible solutions to barriers to reducing children's exposure to ETS. 55 mothers were allocated to the control group and received basic nutritional counselling and brief advice to stop smoking and not expose their children to ETS.

\section{Main outcome measures}

Children's weekly exposure to mothers' tobacco smoke in the home and exposure to tobacco smoke from all sources (mothers' self report and cotinine concentrations from children's urine). Secondary outcomes included smoking cessation, confirmed by cotinine concentrations in the mothers' saliva.

\section{Main results}

Analysis was by intention to treat. Analysis of repeated measures from baseline to 3 months showed that the decline in children's reported exposure to ETS from mothers' cigarettes and from all sources was greater for the counselled group than for the control group $(\mathrm{p}=0.011$ and $\mathrm{p}<0.001$, respectively) and that this was sustained at 12 months $(\mathrm{p}=0.017$ and 0.043 , respectively). Separate analyses for each time point ( 3 and 12 mo) showed that children in the counselled group were exposed to less ETS from their mothers' cigarettes than children in the control group at 3 months (mean $4.5 \mathrm{v}$ 12.1 cigarettes/wk, $\mathrm{p}=0.007$ ), but not at 12 months. Children in the counselled group were exposed to less ETS from all sources at 3 months (13.0 v 26.3 cigarettes/wk, $\mathrm{p}=0.024)$ and 12 months (8.6 $v 19.2$ cigarettes/wk, $\mathrm{p}=0.039)$. Urine cotinine concentrations were similar for children in both groups at 3 months (12.7 $v 13.9 \mathrm{ng} / \mathrm{ml})$, but at 12 months, children in the counselled group had lower cotinine concentrations (10.5 $v 17.5 \mathrm{ng} / \mathrm{ml}$, $\mathrm{p}=0.043)$. The groups did not differ for the number of mothers who stopped smoking ( $6 v 4,\{\mathrm{p}=0.3\} *)$.

\section{Conclusion}

Individualised behavioural counselling for mothers decreased children's exposure to environmental tobacco smoke from all sources at 12 months after counselling. *p Value calculated from data in article.

\section{COMMENTARY}

There is an increasing body of evidence on the health risks for children exposed to ETS. ${ }^{1}$ Although several studies have reported decreased exposure of children to ETS after maternal counselling, ${ }^{2}{ }^{3}$ Hovell et al are the first to supplement self reported tobacco consumption with objective biological markers, measuring cotinine concentrations in both mother and child and conducting nicotine monitoring. The study is further strengthened by ensuring that investigators and laboratory staff were blinded to group assignment. The intensive behavioural counselling intervention was successfully implemented with low income mothers selected from a US supplemental nutrition programme, a sample group arguably less susceptible to anti-smoking health promotion messages. Although most mothers continued to smoke, smoking behaviour was altered to reduce their children's exposure to ETS. Furthermore, a maintenance effect was observed, whereby the reduction of exposure in the counselled mothers was sustained over a 9 month period.

Although the potential benefits of successful ETS reduction programmes are indisputable, the cost of providing individualised counselling was not determined; further work should include cost effectiveness analysis. The costs of individualised counselling of high risk groups are often weighed against the potential benefits that community wide public health messages may bring, particularly when resources are limited. Ideally, comprehensive strategies should incorporate both community based and individual programmes in an attempt to tackle the global tobacco epidemic. ${ }^{4}$ Smoking restrictions and bans are now common within workplace and community settings, yet little attention has been directed at reducing tobacco smoke within the home. Promotion of smoke free rooms or zones within the home will help to reduce the health risks from ETS for young children, who are most vulnerable.

The results are relevant to all healthcare practitioners who interact with pregnant women and young mothers, particularly those within public health or primary care settings. Potential exists for practitioners to provide supportive counselling and education to smoking mothers, highlighting the risk of illness and vulnerability of their children to the effects of ETS.

1 Cook DG, Strachan DP. Thorax 1997;52:1081-94.

2 Greenberg RA, Strecher VJ, Bauman KE, et al.J Behav Med 1994;17:273-90.

3 Hovell MF, Meltzer SB, Zakarian JM, et al. Chest 1994;106:440-6. [Published erratum appears in Chest 1995;107:1480.]

4 Ferrence R, Ashley MJ. BMJ 2000:321:310-11. 